\title{
ANÁLISE DE RESISTÊNCIA À SISTEMA DE INFORMAÇÃO: UMA INVESTIGAÇÃO NO QUADRO FUNCIONAL FEMININO DA UNIVERSIDADE FEDERAL RURAL DA AMAZÔNIA
}

\section{RESISTANCE INFORMATION SYSTEMS ANALYSIS: AN INVESTIGATION INTO THE FEMALE WORKFORCE IN RURAL FEDERAL UNIVERSITY OF THE AMAZON.}

Michelle Borges Miranda ${ }^{1 ;}$ Carlos André Corrêa de Mattos ${ }^{2}$; Ruth Helena Cristo Almeida ${ }^{3}$ Edvar da Luz Oliveira ${ }^{4}$

Universidade de São Paulo ${ }^{1}$; Universidade Federal do Pará ; Universidade Federal Rural da Amazônia ${ }^{3,4}$;

biamichelle@usp.br ${ }^{1}$; carloscmattosail@hotmail.com² ; ruth.almeida@ufra.edu.br ${ }^{3}$; edvar.oliveira@ufra.edu.br';

RESUMO - A Resistência à Sistemas de Informação (RSI) é um fenômeno estudado para entender o comportamento dos usuários de tecnologias quando são submetidos a mudanças de sistemas nas organizações. Tais ações comportamentais podem contribuir negativa ou positivamente dentro da organização, de acordo como o fenômeno é tratado pelos gestores. Entender quais os fatores que levam a ocorrência desse fenômeno nas organizações públicas são importantes para o sucesso organizacional. Este trabalho teve por objetivo identificar e analisar os vetores causadores do fenômeno entre as servidoras UFRA. Nesse sentido adotou-se abordagem quantitativa, utilizando técnicas de amostragem probabilística simples para interpretar os resultados do trabalho de campo.

Palavras-chave: sistemas; resistência; informação.

\begin{abstract}
The Resistance Information Systems (RSI) is a phenomenon studied to understand the behavior of users of technologies systems when they are subjected to changes in organizations. Such behavioral actions can contribute negatively or positively within the organization, according to how the phenomenon is handled by managers. Understanding the factors that lead to the occurrence of this phenomenon in public organization is important to organizational success. This study aimed to identify and analyze the cause of the phenomenon vectors between UFRA servants. In this sense it adopted a quantitative approach, using simple probability sampling techniques to interpret the results of the field work. Keywords: systems; resistance; information.
\end{abstract}




\section{INTRODUÇÃO}

As organizações contemporâneas esperam contar com diversos recursos poderosos em seus Sistemas de Informação (SI), com a finalidade de agilizar o processo de aquisição e tratamento de dados, visando à qualidade da informação. Entretanto, há uma necessidade de organização no gerenciamento dessas informações, e esta coordenação perpassa não apenas por aparatos tecnológicos, ela reflete também na organização de grupos e indivíduos.

O fator humano, bem como seu comportamento, é entendido como uma das principais barreiras na adoção dos $\mathrm{SI}$, sendo até maior que os aspectos técnicos identificados (ROEPKE; AGARWAL; FERRAT, 1998).

Porém, estudos baseados em SI apontam que as organizações estão mais preocupadas com os aspectos de arquitetura de sistemas, principalmente no Brasil, onde se percebe que os enfoques comportamentais e psicológicos são considerados de menor importância. Contudo, embora esta seja uma tendência mundial, internacionalmente houve uma mudança nos estudos, passando de abordagens mais tecnicistas para estudos comportamentais fundamentados nas teorias da psicologia cognitiva e na administração (VAZ E MATTOS, 2011).
A Resistência a Sistema de Informação (RSI) é um fenômeno estudado desde a década de 1980, que tem por objetivo analisar os impactos no comportamento dos funcionários com a implantação ou substituição de Sistema de Informação (KLING, 1980). A RSI é definida como a não utilização, ou utilização indevida, dos SI com o propósito de reduzir ou eliminar o seu uso. Ela é apontada, ainda, como um dos principais fatores de comprometimento do SI e pode ser provocada por diversos motivos pessoais, pelo sistema ou, ainda, por políticas organizacionais que priorizem ou não o investimento em SI, na capacitação dos recursos humanos, entre outros (JOIA E MAGALHÃES, 2009).

Entre os comportamentos observados nos fatores humanos relacionado à RSI, observa-se a manifestação de um processo negativo na implantação e utilização de SI organizacional. Isso porque perdas de poder e ameaças de dimensões têm sido apontadas como umas das causadoras da resistência entre os usuários (KIM E KANKANHALLI, 2009).

A RSI é resultado de uma série de fatores de ordem comportamental, que é manifestado entre os usuários dos sistemas, e podem comprometer seu funcionamento ou aquisição. Inclusive, esse processo pode até mesmo gerar reações de agressividade, 
sabotagem aos sistemas ou simplesmente a ação de não utilização. Do contrário, a aceitação aos sistemas mostra um comportamento oposto, ou seja, os usuários reagem de forma positiva, buscando maximizar seus benefícios.

A implantação de SI em órgãos públicos, como nas universidades federais, foi provocada principalmente pela necessidade de qualidade da informação dada ao gestor para possibilitar a tomada de decisão com menor margem de erro. Além do aperfeiçoamento das práticas de gestão, criação de melhores ambientes de trabalho, entre outros (VAZ E MATTOS, 2011).

A adoção dos SI provoca modificações nas rotinas de trabalho com 0 redimensionamento de tarefas, alterando estratégias corporativas, seja na implantação ou na alteração de procedimento, fato que promove ciclos contínuos de adaptação e readaptação (OLIVEIRA, 2011). E por conta dessas mudanças, vários usuários dos $\mathrm{SI}$ podem desenvolver resistência à implantação e alteração de sistemas.

Outros fatores ganharam destaques no cenário contemporâneo: 0 expressivo investimento nas Tecnologias e Informação e Comunicação, e o aumento da participação feminina das organizações.

$\mathrm{O}$ grande investimento em Tecnologia da Informação e comunicação (TIC) na esfera pública, compreendendo diversas áreas da administração, inclusive em universidades, se dá como forma de melhorar os serviços oferecidos com rapidez e agilidade, evitando desperdício de recursos e de tempo, para tornar o serviço público mais eficiente e ampliar suas relações de negócios.

Também, os estudos sobre mulheres não são tidos mais como restrito no campo das reivindicações políticas, mas passou a ser considerado como um fator essencial no desenvolvimento econômico e social. Observa-se que houve um aumento expressivo no quadro do funcionalismo público de mulheres. O número de servidoras, por exemplo, do sexo feminino chega a $45,6 \%$ do total de servidores do país, e só na educação alcançaram $48,9 \%$ do total de servidoras segundo o último boletim estatístico de pessoal de 2012 (BRASIL, 2012). No setor informal de empregos, as mulheres sempre foram a máxima maioria durantes anos, e paulatinamente através dos séculos vem ocupando seus espaços no setor formal.

Embora a resistência possa ocorrer com qualquer usuário, esse aspecto torna ainda mais relevante estudar o fenômeno na contemporaneidade, onde a participação feminina nas organizações assume destaque progressivo. Neste sentido, o estudo serve como estratégia para combater os aspectos negativos da RSI, reduzir características prejudiciais e fortalecer a adoção aos 
sistemas, bem como identificar e tomar ações que possam minimizar a RSI, analisando, em especial a participação feminina dentro das organizações. Uma vez que, as organizações na busca por maximizar seus serviços, estão investindo cada vez mais em alta tecnologia, nos Sistemas de Informações, e no melhoramento dos recursos humanos.

Por isso, entender o fenômeno da RSI e quais fatores influenciam para acentuá-lo dentro de uma organização pode contribuir para o desenvolvimento de estratégias de combate dos aspectos negativos, e para melhorar o processo de adoção dos sistemas. Uma vez que, as organizações na busca por maximizar seus serviços, estão investindo cada vez mais em alta tecnologia, nos Sistemas de Informações e no melhoramento dos recursos humanos. Portanto, o objetivo desse estudo é identificar e analisar as variáveis indicadoras do fenômeno denominado Resistência a Sistema de Informação entre as servidoras da Universidade Federal Rural da Amazônia.

\section{FUNDAMENTAÇÃO TEÓRICA}

\subsection{SISTEMAS DE INFORMAÇÃO NAS INSTITUIÇÕES PÚBLICAS DE ENSINO}

A administração contemporânea, e em particular a administração pública, estão se utilizando cada vez mais das Tecnologias da Informação e dos SI automatizados, em todos os níveis da administração pública tanto na esfera federal, quanto estadual e municipal. Isso porque a transformação da economia industrial para economia da informação torna os SI fundamentais para as organizações. Desafiando os servidores públicos para constante objeto de superação, uma vez que, esses sistemas assim como o hardware e a TC são alvos de constantes mudanças, transformações e inovações, numa velocidade surpreendente.

$$
\text { Laudon e Laudon (1999) definem }
$$
sistema de informação como função coletar, recuperar, processar, armazenar e distribuir informações, tendo por finalidade agilizar o processo de organização, planejamento, controle, coordenação, análise e decisão dentro de organizações. Esses sistemas, segundo Alter (1996), se utilizam das Tecnologias de Informação em um ou mais processos de negócios.

As instituições de ensino também se utilizam dos SI com objetivo de integralizar a área administrativa com a acadêmica, proporcionando assim uma gestão e controle dos desperdícios, orçamento, podendo com isso oferecer maior visibilidade e transparência dos processos de decisão. Muito embora, a importância dos SI seja muito mais perceptível nas organizações empresarias que a enxergam como forte aliada na competitividade e na busca por vantagens no mercado, as organizações públicas ainda enfrentam grandes 
dificuldades em adotá-las, e com consequência de um conjunto de fatores, entre eles a velocidade do ambiente competitivo a fim de regular ou oferecer serviços à sociedade (CUNHA et al., 2011).

Acredita-se que o maior impedimento na adoção de tecnologias e SI nas organizações públicas brasileiras está numa barreira burocrática e cultural, que impede a agilidade dos processos internos. Cunha et al. (2011) afirmam que na maioria das instituições públicas de ensino é inexistente o controle eficiente do fluxo de informação, por parte dos dirigentes, em termos de utilização otimizada dos recursos destinados pelo Governo Federal. Isso se apresenta como consequência do investimento em $\mathrm{TI}$ suas prioridades nas divisões de investimentos.

Percebe-se um processo, entretanto, nas instituições de ensino tecnológico, principalmente melhoras a partir de 1996, quando as escolas técnicas passaram para centros de educação tecnológica, e mais recentes em 2008 com a transformação destes em Institutos Federais de Educação, Ciência e Tecnologia. Muito embora, este processo de adoção aos SI seja uma resposta à alteração da missão, dos conceitos e valores das instituições, como forma de utilizar os escassos recursos de forma eficiente.
Porém, para Almeida (2000) apesar de algumas IES brasileiras estarem em conformidade com os padrões modernos de administração, a renovação é baixíssima, tornando a maioria das instituições vinculadas a modelos burocráticos e ultrapassados com quase nenhuma visão estratégica.

Entretanto, as IES nunca estiveram alheias aos avanços da TI. Na década de 1930 a postura delas em relação à informática, por exemplo, era passiva sempre no aguardo de novas demandas de usuários. Sendo adotada gradativamente com os avanços da TI tanto na área acadêmica como na área administrativa, embora a incorporação ocorra de forma descontínua e inadequada, resultando em problemas e resistência (ALBERTINI, 2001).

\subsection{A PARTICIPAÇÃO FEMININA NO MERCADO DE TRABALHO E A ATUALIDADE NO SERVIÇO PÚBLICO}

Segundo o Boletim Estatístico de Pessoal do Ministério do Planejamento e Gestão, a participação feminina no funcionalismo público tem crescido em vários setores, tendo uma participação em média de $45 \%$ no quadro funcional total dos serviços públicos no país, embora essa percentagem tenha se mantido quase estável 
durante o período de 10 anos, entre 2002 e $2012^{1}$.

Merece destaque que Olinto e Oliveira (2006) já apontava para o aumento e as mudanças nas relações de trabalho feminino no Brasil, demonstrando que a participação feminina vinha crescendo nas ocupações do setor formal, embora esse crescimento ainda fosse significativamente menor quando se comparado ao crescimento masculino nas ocupações no setor formal.

A autora destaca que historicamente as mulheres tendem a ser maioria nos setores informais de trabalho, ou ainda em trabalhos considerados precários, ou seja, aqueles onde não se tem perspectiva de carreira, onde os direitos trabalhistas são poucos ou completamente ignorados. A situação de discrepância é ainda maior quando os dados de participação feminina são analisados dentro dos setores de Ciência e Tecnologia.

Embora a participação das mulheres esteja crescendo substancialmente de maneira geral nas ocupações de Ciência e Tecnologia, nas áreas mais específicas de TI tal participação ainda é mínima. Esse fato ocorre, de acordo com Olinta e Oliveira (2005), por que existe uma cultura feminina imposta às meninas desde a infância sobre a relação de trabalho, e as áreas ditas como

\footnotetext{
1 Optou-se pelos relatórios de gestão de 2012 na maioria dos casos, por identificarmos como um dos últimos maiores períodos de contratação de servidores.
}

tipicamente femininas. Isso é verificado nas relações familiares, levado à escola, fazendo com que as meninas em sua vida acadêmica se tornem mais tímidas desde a escolha de um curso de nível superior, até o simples fazer acadêmico como a utilização de uma ferramenta computacional. E é levado para a vida profissional também, transformando-se numa barreira para novos aprendizados e elevação de carreira.

É claro que esta realidade tende a mudar com a informação, educação integralizada e interdisciplinar. Já que, segundo o último Censo (IBGE, 2010) as mulheres tendem a ter o maior nível acadêmico, uma vez que estas passam mais tempo nas escolas. Na pós-graduação o número de mulheres já chega à metade das vagas preenchidas nos mais diversos setores, com exceção dos cursos específicos de $\mathrm{TI}$ como Sistema de Informação, Ciência da Computação e Engenharia da Computação.

Entretanto, é necessário frisar que o estudo de gênero nas organizações não se limita mais ao campo político de tema. Cada vez mais assumem status nas questões econômica e social, uma vez que a participação da mulher tem crescido e contribuído cada vez mais para o desenvolvimento dos diversos setores da economia, educação, entre outros. Por isso, estudar os fatores que as impeçam de alavancar neste processo, de forma a 
contribuir com o melhor desenvolvimento das mesmas dentro das organizações, é fundamental para gestores e para a melhor implantação dos SI dentro da organização, bem como identificar os fatores de resistência para que tal fenômeno aconteça.

\subsection{RESISTÊNCIA AOS SISTEMAS DE INFORMAÇÃO}

Comumente as pessoas tendem a agir com resistência ao novo, quando as mesmas se sentem ameaçadas. Para Schneider e Reichers (1983), as pessoas naturalmente resistem a mudanças. Diversos autores que tratam sobre RSI, tal como Markus (1983) e Joia (2006), também entendem que os motivos pessoais tais como a aptidão com tecnologias, falta de experiência com SI, por exemplo, podem levar o usuário a desenvolver um processo de resistência. Segundo Markus (1983) existem várias explicações para que tal fenômeno aconteça, dentre eles, a simples resistência à mudança, como também quando se percebe que os custos para que alguma mudança aconteça é superior aos benefícios provocados pela mesma.

A resistência à mudança teve seu primeiro estudo empírico realizado por Coch e French (1948), os quais influenciaram fortemente as pesquisas na década de 1950 e 1960. O tema sofreu um relativo silêncio na década de 1970 e 1980 e só voltou a ser discutido com grande ênfase novamente a partir da década de 1990 (CALDAS E HERNANDEZ, 2001).

Curiosamente, na década de 1990, um fenômeno chamado de RSI começa a ser tratado e publicado em artigos, tendo como principais autores: Kling (1980), Markus (1983), Joshi (1991), Marakas e Hornik (1996), Martinkoet et al. (1996), Lapointe e Rivard (2005) e Joia (2006).

Mas, recentemente, tem-se um trabalho abrangente que reuniu os principais aspectos das teorias, com a proposta de estruturar um meta-modelo que, segundo o autor Gaete (2010), incorpora os aspectos da dinâmica e recursividade do relacionamento entre o agente - usuário do sistema de Informação - e o sistema propriamente dito, no seu contexto de uso.

A Resistência a Sistema de Informação pode ser definida segundo Markus (1983) como a oposição dos usuários a uma mudança percebida e relacionada à introdução de um novo sistema. Cabe ao implementador da mudança responder abertamente essa expressão de resistência, buscando superá-la (MARKUS, 1983).

Não obstante, Lapointe e Rivarde (2005) abordam comportamento de resistência, classificando-o em quatro principais grupos: apatia - relacionada à distância e à falta de interesse; resistência pacífica - relacionada a táticas de demora, abandono, desculpa e persistência em 
comportamentos antigos; resistência ativa relacionada a comportamento forte, não destrutivo e formação de coalizões; e resistência agressiva - que abrange ameaças, brigas, boicotes ou sabotagens procurando acabar com a ordem ou destruir.

Porém, Markus (1983) afirma ainda que quando o objeto de estudo se fundamenta na resistência, um olhar cuidadoso deve ser estabelecido. Não é incomum a associação de resistência com má utilização ou não utilização de um sistema ou de um serviço. Pois, para a autora, pode haver vários motivos que causam resistência do indivíduo ao sistema, como a ignorância na utilização, a resistência à mudança, ou o sistema não ser necessário para sua utilização, e que este último em particular não deve ser considerado como resistência. $A$ autora destaca ainda que a resistência possui aspectos negativos e positivos.

Analisando o trabalho de Kling (1980) e suas teorias sobre resistência desenvolvidas, Markus (1983) verificou três vetores vindos das teorias de Kling (1980), das quais ele analisou aspectos da computação na vida organizacional, com a introdução de SI computacional e resistência humana, o que na análise dele quase sempre se faz presente.

Os estudos e desenvolvimentos de pesquisas sobre análise de RSI avançaram principalmente no campo individual e comportamental dos usuários. Entretanto, poucos estudos se tornaram tão abrangentes, no escopo quanto os estudos de Markus (1983) pelo tratamento aprofundado dos aspectos organizacionais relativos à introdução de sistema de informação (JOIA E MAGALHÃES, 2009).

O exemplo disto, o modelo teórico de Joshi (1991), no qual a resistência é analisada em relação ao status de equidade trazido pelo sistema de informação ao indivíduo, em comparação a sua situação antes da implementação do sistema, analisando se houve uma desvantagem significativa em relação ao seu status inicial, ou menor em relação aos outros indivíduos da organização.

Já Marakas e Hornik (1996) tratam do comportamento como resposta passiva ou agressiva por conta da implantação do sistema de informação. Esse comportamento para os autores advém de características pessoais e incertezas diante da chegada de um novo sistema, que resultam em uma forma mascarada de aceitação, mas que, entretanto, resulta no uso inadequado do sistema consciente.

Segundo Martinko, Henry e Zmud (1996), a aceitação ou resistência às Tecnologias da Informação são dadas por um conjunto de fatores relacionados, que compreende o perfil e as características do indivíduo, na experiência prévia com tecnologias semelhantes, variáveis internas e 
externas à organização, que somadas influenciam nas expectativas e nos resultados futuros.

Lapointe e Rivard (2005) apresentam novamente uma primeira iniciativa, após Markus (1983), ao estudarem a resistência de maneira individual e coletiva, agrupada. Desta forma, propuseram um modelo multinível, individual e de grupo, baseado nos trabalhos anteriores a resistência, identificando assim cinco componentes da mesma: (1) comportamento, (2) objeto de resistência, (3) sujeito, (4) ameaças e (5) condições iniciais. As autoras observaram que o nível de resistência varia do individual para o coletivo, do técnico para o político, conforme o avanço e o tempo da implantação do sistema de informação, para isso as autoras analisaram grupos a partir de indivíduos.

Para Lapointe e Rivard (2005) resistências às mudanças ocorrem basicamente pelos mesmos motivos, que alguns outros autores destacaram a alteração das condições iniciais, ameaças individuais, e de grupos, por conta da nova reorganização. Entretanto para Joia e Magalhães (2009), as autoras Lapointe e Rivard (2005) mesmo tendo sido dinâmicas relacionando resistência há variável tempo que se é implantando e estudando novamente as questões individuas e coletivas, os autores consideram o estudo pouco aprofundado nas questões de organizações, relativas à implantação de sistema de informação.

Este trabalho considera Resistência a Sistema de Informação alinhada com as definições expostas de Kling (1980) e Markus (1983), e que Gaete (2010) definiu como ação - ou falta de ação-, intencional, que se opõe ou ignora a implantação de um sistema de informação, podendo se manifestar de diversas intensidades ao longo do tempo, e tem por base o modelo proposto por Gaete (2010), modelo de cinco vetores: (1)Propriedades Institucionais, (2) Sistemas, (3) Integração Sócio-técnica, (4) Política e (5) Pessoas.

Figura 1. Modelo proposto por Gaete (2010).

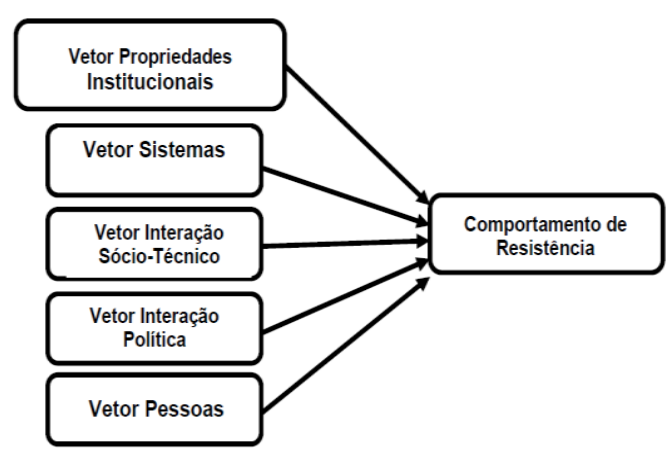

Fonte: (Gaete, 2010).

\section{METODOLOGIA}

Este estudo utilizou procedimentos metodológicos classificados como exploratórios e descritivos, com abordagem quantitativa para o tratamento dos dados (MARCONI E LAKATOS, 2008).

O universo desta pesquisa foi composto por servidoras públicas, que trabalham na UFRA e utilizam ou utilizaram 
SI. O cálculo para a quantificação da amostra foi realizado considerando os dados disponíveis no Relatório de Gestão da Universidade (UFRA, 2012), onde está informado o total de mulheres da organização (207 mulheres) e suas áreas de atuação. A técnica de amostragem utilizada foi probabilística aleatória simples, que de acordo com Hair et al. (2009) é um “método direto de amostragem que atribui a cada elemento da população-alvo uma igual probabilidade de ser selecionado".

O cálculo da amostra foi realizado conforme Pinheiro et al. (2004) que utilizaram a fórmula para cálculos de universos finitos envolvendo proporções (Figura 2); foram considerados os seguintes parâmetros para a quantificação da amostra: (1) margem de segurança 95,5\% (2 desviospadrões); (2) margem de erro 5\%. A amostra resultou em 137 entrevistados, quantidade obtida de questionários válidos após pesquisa de campo.

Figura 2. Formula ${ }^{2}$ para cálculo de universo finitos

$$
n=\frac{0,25 * Z^{2} * N}{0,25 * Z^{2}+e^{2} *(N-1)}
$$

Fonte: (Pinheiro et al., 2004).

\footnotetext{
$2 \mathrm{~N}$ = tamanho do universo; $\mathrm{n}=$ tamanho da amostra; S 2 = variância estimada da amostra; e= erro amostral; $\mathrm{Z}=$ desvio-padrão relacionado ao índice de confiança.
}

Os dados foram coletados em pesquisa de campo por meio de entrevistas pessoais. A forma utilizada para a coleta de dados foi o questionário estruturado. Vergara (2013) ao explicar questionário apresenta-o como uma série ordenada de perguntas fechadas, semiabertas e de múltipla escolha. Para analisar empiricamente a RSI o questionário de pesquisa foi adaptado de Gaete (2010).

A pesquisa de campo ocorreu no período de 01 de novembro de 2014 a 29 de março de 2015, o longo período deu-se em razão das dos feriados comemorativos de final e início de ano. Os dados foram coletados no campus da UFRA, localizados em Belém, Capitão Poço, Paragominas e Parauapebas, todos no Estado do Pará.

Neste período, foram aplicados 137 questionários de pesquisa, todos considerados válidos após avaliação preliminar. Participaram da pesquisa servidoras, independente de cargos e funções que ocupavam na instituição.

No perfil das entrevistadas, observouse que a maioria possui entre 35 a 45 anos de idade que corresponde a 45 mulheres $(33,3 \%)$. Esse primeiro grupo somado ao segundo grupo com maior frequência, aquele entre 25 a 35 anos de idade, compõem a maioria das entrevistadas (53,5\%).

O tempo de experiência com SI também foi analisado de acordo com as 
informações obtidas através da pesquisa de campo. A maioria das entrevistadas, 50 mulheres (36,2\%), possui entre 4 e 7 anos de experiência com Sl; percebemos ainda que 12 mulheres entrevistadas $(8,7 \%)$ possuem acima de 12 anos de experiência com SI, o que torna, pela experiência acumulada das entrevistadas, as respostas mais representativas.

Entre as entrevistadas, observou-se que $01(0,7 \%)$ não possui nenhuma experiência com SI. Entretanto, de acordo com as anotações de campo, quando questionadas sobre implantação e mudanças trazidas pelos SI ela reconhece a importância, utilidade e necessidade do mesmo, mas ressalta que existe uma carência de incentivos a formação técnica e tecnológica para funcionários não ligados diretamente aos departamentos de TI.

O grau de escolaridade também foi observado, percebeu-se que 97 mulheres (71\%) possuem nível superior sendo, na maioria docentes, o segundo grupo com maior frequência foi de assistentes administrativas. Observou-se ainda que 02 mulheres $(1,4 \%)$ possuem apenas nível fundamental.

O questionário aplicado foi elaborado em duas seções, a primeira reuniu perguntas fechadas e semiabertas que foram selecionadas para caracterizar os entrevistados.
A segunda parte do instrumento foi elaborada em afirmativas mensuradas por escala intervalar de Licket com 5 opções de respostas que variaram entre (1) discordo completamente; (2) discordo em parte; (3) nem discordo nem concordo; (4) concordo em parte e; (5) concordo completamente. A investigação específica do fenômeno iniciou no questionário com afirmativas que avaliaram a predisposição do entrevistado para desenvolver resistência ou aceitabilidade aos SI; em seguida as afirmações passaram a envolver aspectos das cinco dimensões do construto que foram apresentadas na seção Revisão de Literatura, e formaram os vetores: (1) pessoas; (2) sistemas; (3) sócio-técnica; (4) política; (5) propriedades institucionais (GAETE, 2010).52 Os dados foram tratados de forma quantitativa, na primeira fase cada pergunta foi enumerada e repassada para planilha eletrônica. As respostas dos entrevistados após codificação sequencial seguiram o mesmo processo. Essa etapa foi necessária para a formação da matriz que possibilitaria o tratamento dos dados e a realização das análises matemáticas e estatísticas. As variáveis foram agrupadas de acordo com os construtos, denominados como vetores segundo a teoria da RSI. Para interpretar os resultados foi necessário elaborar indicadores que foram calculados, tanto para 
as variáveis quanto para os entrevistados, de acordo com a seguinte formula:

Figura 3. Calculo ${ }^{3}$ dos indicadores

$$
\begin{aligned}
& \text { Índice }=\underline{\left(\sum D T^{*} 0\right)+\left(\sum D P^{*} 0,25\right)+\left(\sum \text { indeciso* } 0,50\right)+\left(\sum C P^{*} 0,75\right)+\left(\sum C T^{*} 1\right)} \\
& \text { Número de entrevistados }
\end{aligned}
$$

Fonte: (Pinheiro et al., 2004).

Os indicadores são expressos na forma de índices e puderam assumir valores entre 0,0000 (zero) e 1,000 (um) para expressar a intensidade da variável, do vetor e do entrevistado em relação à RSI. A interpretação dos índices ocorreu de acordo com os valores obtidos. Os índices abaixo de 0,4000 foram considerados como indicativos da presença de RSI, valores entre 0,4001 e 0, 6999 interpretados como intermediários e valores iguais ou superiores a $0,7000 \mathrm{com}$ aceitação aos SI.

A utilização de indicadores destaca-se neste estudo por sua capacidade em transformar atributos qualitativos em quantitativos e assim favorecer o tratamento dos dados com técnicas estatísticas e matemáticas, além de possibilitar a análise de cada variável dentro do vetor e dos vetores dentro do construto.

Os vetores tiveram a consistência interna testada pelo coeficiente alpha de Cronbach ( $\alpha$ ). A forma de cálculo é apresentada por Rodrigues e Paulo (2009) e representado na Figura 4, o alpha de Cronbach varia entre 0,000 (zero) e 1,000

\footnotetext{
${ }^{3}$ DT é discordo totalmente; DP é discordo parcialmente; CP é concordo parcialmente; CT é concordo totalmente.
}

(um). Desta forma, quanto mais o índice se aproxima da unidade mais confiáveis são as respostas. Hair et al. (2009) destacam que valores superiores a 0,600 evidenciam confiabilidade de medidas.

Figura 4. Alpha ${ }^{4}$ de Cronbach

$$
\alpha=\frac{\mathrm{N}}{\mathrm{N}-\mathbf{1}}\left(1-\frac{\sigma \sum_{\mathrm{y}}^{2}}{\sigma_{\mathrm{x}}^{2}}\right)
$$

Fonte: (Pinheiro et al., 2004).

Para a avaliação do grau de RSI entre grupos de entrevistados, os índices médios foram calculados e os entrevistados posicionados considerando suas distâncias da média. Assim, diferenças superiores ou inferiores a 1, desvio-padrão, foram consideradas como indicativos de aceitabilidade ou RSI. Esse procedimento possibilitou identificar o grau de RSI entre os entrevistados e fundamentou a formação de agrupamentos.

\section{RESULTADOS E DISCUSSÕES}

De acordo com a teoria da RSI, segundo o modelo teórico proposto por Gaete (2010), existem cinco vetores de RSI: pessoa, sistemas, sócio-técnico, político e propriedades institucionais.

As questões que compõem o questionário foram agrupadas de acordo com cada vetor e formaram seis grupos capazes de sistematizar processos em resistência. A

\footnotetext{
${ }^{4}$ Em que: $\mathrm{N}$ é o número de itens do questionário; $\sigma$ y2 é a variância do item i;
} 
análise da RSI iniciou com uma avaliação geral para observar uma predisposição da entrevistada para desenvolver processos de resistência. Assim, foi analisado à disposição das entrevistadas pela resistência ou aceitabilidade aos SI. As questões foram agrupadas de forma a perceber a adaptação aos SI. O alpha de Cronbach obtido foi calculado em 0,730 , considerado como aceitável para pesquisas exploratórias.

Sobre a resistência e aceitabilidade dos sistemas, resultados indicam que as servidoras da UFRA demonstram menor RSI, e estão dispostas às mudanças, por entenderem que os sistemas trazem transformações positivas no que se refere a SI. Entretanto, a cooperação na implantação de SI foi avaliada como intermediária $(0,6449)$ na Tabela 1.

Tabela 1. Resistência ou aceitabilidade de Sistemas de Informação.

\begin{tabular}{ll}
\hline Variável & Índice \\
\hline $\begin{array}{l}\text { Adapto-me facilmente às mudanças trazidas } \\
\text { pelos Sistemas de Informação. }\end{array}$ & 0,7808 \\
$\begin{array}{l}\text { Coopero com a implantação dos Sistemas de } \\
\text { Informação e novas maneiras de trabalhar. }\end{array}$ & 0,6449 \\
$\begin{array}{l}\text { Concordo com a implantação e mudanças } \\
\text { trazidas pelos Sistemas de Informação. }\end{array}$ & 0,8424 \\
$\begin{array}{l}\text { De maneira geral não resisto à utilização de } \\
\text { Sistemas de Informação. }\end{array}$ & 0,8623
\end{tabular}

Alpha de Cronbach $=0,730$.

Fonte: (Pesquisa de campo, 2015).

No vetor pessoas foram agrupadas as questões associadas às características individuais e atitudes, como destaca Markus (1983). Essas características foram relacionadas com histórico pessoal com $\mathrm{SI}$, experiências, motivações e interesses que podem indicar predisposição pessoal ao uso de SI e da Tecnologia de Informação de uma forma geral. Neste aspecto o interesse pessoal, como destaca Rocha e Ghoshal (2006), é uma forma de maximizar os benefícios do sistema através de uma escolha racional do usuário (MACEDO, 2011). A Tabela 2 apresenta o resultado do vetor e o valor dos indicadores. O alpha de Cronbach obtido foi calculado em 0,647 , considerado como aceitável, após a análise das respostas das entrevistadas.

A Tabela 2 demonstra que em geral as mulheres entrevistadas não possuem RSI, entretanto, também não indica que há uma forte aceitabilidade sobre os sistemas quanto ao vetor pessoas. Isso pode ser observado nos índices resultantes de cada variável, onde os resultados sugerem que as experiências advindas de outros sistemas $(0,8732)$ facilitam a adaptação a novos sistemas, facilitando no dia a dia $(0,8859)$, aumentando a produtividade (0,9529). A busca por tecnologias e a aptidão pessoal $(0,9094)$ influenciaram positivamente na análise. Porém, os resultados apontam um índice baixo indicando que há necessidade de esforços para a persuasão e treinamento para a utilização dos sistemas $(0,5996)$ e quanto à interface com o usuário $(0,6413)$.

Tabela 2. Vetor pessoas na Resistência a Sistema de Informação. 


\begin{tabular}{|c|c|}
\hline Variável & Índice \\
\hline $\begin{array}{l}\text { Minha experiência com Sistemas de } \\
\text { Informação facilitam a adaptação a } \\
\text { novos sistemas e tecnologias }\end{array}$ & 0,8732 \\
\hline $\begin{array}{l}\text { Busco conhecer os recursos disponíveis } \\
\text { no sistema para melhorar meu trabalho } \\
\text { diário }\end{array}$ & 0,9094 \\
\hline $\begin{array}{l}\text { Gosto de tecnologia, de conhecer o que } \\
\text { há de novo na área e de incorporar } \\
\text { avanços tecnológicos no meu dia a dia. }\end{array}$ & 0,7880 \\
\hline $\begin{array}{l}\text { Os Sistemas de Informação que utilizo } \\
\text { atualmente na organização facilitam } \\
\text { meu dia a dia }\end{array}$ & 0,8859 \\
\hline $\begin{array}{l}\text { Considero os Sistemas de Informação } \\
\text { que utilizo simples e fáceis de serem } \\
\text { utilizados desde o início }\end{array}$ & 0,6413 \\
\hline $\begin{array}{l}\text { Noto que alguns colegas se sentem } \\
\text { ameaçados pela implementação de } \\
\text { Sistemas de Informação }\end{array}$ & 0,7789 \\
\hline $\begin{array}{l}\text { De maneira geral a produtividade } \\
\text { melhorou com a utilização de Sistemas } \\
\text { de Informação }\end{array}$ & 0,9529 \\
\hline $\begin{array}{l}\mathrm{Na} \text { organização que trabalho há } \\
\text { necessidade de muito treinamento e } \\
\text { persuasão para que os Sistemas de } \\
\text { Informação } \\
\begin{array}{l}\text { sejam } \\
\text { corretamente }\end{array}\end{array}$ & 0,5996 \\
\hline $\begin{array}{l}\text { Considero que a utilização de Sistemas } \\
\text { de Informação trouxe (ou trará) } \\
\text { mudança de status para alguns dentro } \\
\text { da organização }\end{array}$ & 0,7446 \\
\hline
\end{tabular}

Alpha de Cronbach $=0,647$.

Fonte: (Pesquisa de campo, 2015).

As características dos sistemas podem contribuir para a geração de atitudes favoráveis ou negativas, representadas pela má utilização dos SI ou mesmo pela recusa total de uso. Este comportamento pode ser reforçado se houver ineficiência do sistema, seja pela dificuldade ou complexidade de utilização do sistema, ou mesmo por características técnicas, falhas, limitações de funcionalidade, desempenho ou ainda interface (MARKUS, 1983; JOIA, 2006). As variáveis utilizadas para mensurar o vetor sistema estão descritas na Tabela 04. O alpha de Cronbach foi calculado em 0,880 é considerado como bom, demonstrado a representatividade dos resultados.

Das sete variáveis agrupadas no vetor sistema, conforme a Tabela 3, seis apresentaram índices intermediários, entre 0,4000 a 0,6999. Foram elas representadas por aspectos como visualmente agradáveis $(0,5543)$, fáceis de utilizar $(0,5707)$, atendem minhas necessidades $(0,5308)$, rapidez e desempenho $(0,4529)$, relatórios e consultas $(0,4873)$, adaptabilidade e flexibilidade $(0,4855)$ e em avaliação geral $(0,7409)$. Esses aspectos fornecem insight quanto ao que pode ser melhorado no vetor de forma a evitar processo de resistência melhorando o retorno dos investimentos em SI.

Tabela 3. Vetor sistemas na Resistência a

\begin{tabular}{ll}
\hline Variável & Índice \\
\hline $\begin{array}{l}\text { Os Sistemas de Informação que utilizo são } \\
\text { visualmente agradáveis }\end{array}$ & 0,5543 \\
$\begin{array}{l}\text { Os Sistemas de Informação que utilizo são } \\
\text { fáceis de utilizar }\end{array}$ & 0,5707 \\
$\begin{array}{l}\text { Os recursos disponíveis nos sistemas que } \\
\text { utilizo atendem as minhas necessidades }\end{array}$ & 0,5308 \\
$\begin{array}{l}\text { Os sistemas de informações que utilizo } \\
\text { são rápidos e têm boa performance }\end{array}$ & 0,4529 \\
$\begin{array}{l}\text { Os relatórios e consultas são adequados e } \\
\text { suficientes para atender-me }\end{array}$ & 0,4873 \\
$\begin{array}{l}\text { Os sistemas escolhidos pela organização } \\
\text { são flexíveis e se adaptam rapidamente a }\end{array}$ & 0,4855 \\
novas necessidades dos usuários & \\
$\begin{array}{l}\text { No geral, os Sistemas de Informação } \\
\text { utilizados pela organização que trabalho }\end{array}$ & 0,7409 \\
$\begin{array}{l}\text { atende a forma de trabalhar da empresa e } \\
\text { as nossas necessidades }\end{array}$ & \\
\hline
\end{tabular}

Sistema de Informação.

Alpha de Cronbach $=0,880$.

Fonte: (Pesquisa de campo, 2015).

O vetor interação sócio-técnico está

relacionado com a interação do sistema com a distribuição do trabalho e das 
responsabilidades (MARKUS, 1983). De acordo com a Tabela 4, quando analisadas as variáveis, percebeu-se que as entrevistadas apresentaram dificuldades na adaptação do sistema de informação quanto às rotinas da instituição, quando há mudanças, havendo esforço para ajustar os modos de trabalho ao sistema.

Destaca-se também que o jeito de trabalhar continua o mesmo $(0,8116)$. Essa resposta indica uma subutilização dos sistemas, uma vez que, como eles não são completamente ajustadas as reais necessidades dos usuários, eles ficam limitados e restritos às atividades essenciais não chegando a alterar o processo de trabalho.

O alpha de Cronbach, calculado para este vetor, resultou em 0,054 , considerado inaceitável. Este resultado é decorrência da variação dos resultados dos índices das variáveis agrupadas, onde ocorreram índices abaixo de 0,500 e acima de 0,800, demonstrando uma baixa consistência interna nas respostas das entrevistadas. Desta forma, pode-se observar que, embora as entrevistadas não apresentem ações de RSI, como ação para destruir os sistemas, elas também não apresentam aceitabilidade alta, contribuindo com a incorporação de suas rotinas de trabalho no mesmo.

Observa-se também que as redistribuições de tarefas e responsabilidades ocasionadas pelos SI podem ser em consequências das recentes mudanças ocorridas na Instituição, na transformação da estrutura organizacional da Instituição. Este vetor, assim como os demais, pode desencadear processos RSI quando causam grandes mudanças nas rotinas de trabalho, ou quando muda a estrutura organizacional, alterando a estrutura de poder e tarefa dentro da organização (MARKUS, 1983).

\begin{tabular}{|c|c|}
\hline Variável & Índice \\
\hline $\begin{array}{l}\text { Considero que as rotinas de trabalho são } \\
\text { compatíveis com os Sistemas de } \\
\text { Informação utilizados pela organização, } \\
\text { não sendo necessário um grande esforço } \\
\text { para ajustar o modo de trabalhar ao } \\
\text { sistema utilizado na organização }\end{array}$ & 0,4801 \\
\hline $\begin{array}{l}\text { Os Sistemas de Informação utilizados na } \\
\text { organização trazem uma redistribuição } \\
\text { de responsabilidades e de trabalho }\end{array}$ & 0,4909 \\
\hline $\begin{array}{l}\text { Apesar da utilização de Sistemas de } \\
\text { Informação o "jeito" de trabalhar } \\
\text { continua o mesmo }\end{array}$ & 0,8116 \\
\hline
\end{tabular}

Tabela 4. Vetor sócio-técnico na Resistência a Sistema de Informação.

Alpha de Cronbach $=0,054$.

Fonte: (Pesquisa de campo,2015).

No vetor político, o alpha de Cronbach foi calculado em 0,828 , sendo considerado muito bom. O vetor interação política analisou a percepção de mudança no status de pessoas dentro da organização em decorrência da utilização de SI, bem como a consolidação ou despontamento de grupos em decorrência dos SI e, a alteração de poder, tal como prever Joia (2006), foi percebido entre as entrevistadas. 
Importante ressaltar que os aspectos políticos de acordo com Lapointe e Rivard (2005) são considerados como uma etapa para evolução do comportamento de Resistência a SI, que indicam a transição de aspectos puramente técnicos para questões políticas para a implantação e uso de um novo sistema (GAETE, 2010).

Posto isto, foi observado na Tabela 5 que há uma tendência à Resistência a Sistema de Informação em decorrência de aspectos políticos, ou em decorrência de disputa interna na organização, na consolidação ou despontamento de grupos em razão dos SI. Desta forma, esse vetor foi o que apresentou maior tendência a processos de RSI, pois apesar de posicionar-se ainda na faixa intermediária a avaliação geral do vetor foi o que apresentou a menor pontuação geral (Figura 05). Dentre as variáveis desse vetor destacam-se os aspectos relacionados à distribuição de poder $(0,4728)$, consolidação de grupos $(0,5471)$ e processos de disputas internas $(0,5344)$.

Tabela 5. Vetor política na Resistência a Sistema de Informação.

\begin{tabular}{ll}
\hline Variável & Índice \\
\hline Os Sistemas de Informação implantados na & 0,4728 \\
organização ocasionaram uma redistribuição & \\
de poder & \\
Observo que politicamente um indivíduo ou 0,5471 \\
grupo de indivíduos se consolidou ou \\
despontou como decorrência da utilização de \\
Sistemas de Informação \\
Observo que ocorrem disputas políticas 0,5344 \\
internas que dificultam a utilização dos \\
Sistemas de Informação \\
\hline Alpha de Cronbach $=0,828$.
\end{tabular}

Fonte: (Pesquisa de campo,2015).

Já no vetor propriedades institucionais o objetivo mais geral é analisar os processos, procedimentos, regras e normas mais formais dos procedimentos propriedades institucionais, a análise do perfil de seus líderes e a própria cultura da organização que são as regras aceitas e internalizadas pela organização. Estas regras e recursos, de acordo com Gaete (2010), Orlikowski e Robey (1991), são utilizados pelos agentes humanos e mediam suas ações diariamente, tanto para facilitar quanto para constranger as mesmas na organização, que poderá influenciar no comportamento de RSI. O alpha de Cronbach calculado foi de 0,730, considerado bom.

O vetor Propriedades Institucionais, proposto por Gaete (2010), agrupam-se variáveis relativas também ao ambiente de Tecnologia da Informação. De acordo com essas variáveis, a cultura de organização, o estilo de liderança, as normas de implantação e utilização de SI, investimento em soluções tecnológicas são apontadas como intermediárias pelas usuárias dos sistemas, ou seja, posicionaram-se com resultados entre 0,4001 e 0,6999. Porém destaca-se a variável de Tecnologias de Informação, o índice posicionou-se como baixo na escala utilizada (0,3641), evidenciando que a instituição é tecnologicamente atrasada e investe pouco nesse aspecto segundo a 
opinião das entrevistadas. Essa resposta é um indicativo que sugere risco de processos de resistência, pois o surgimento de novas e melhores tecnologias não é acompanhado pela Instituição. As demais variáveis posicionaram-se na faixa intermediária, conforme se observa na Tabela 6.

Tabela 6. Vetor Propriedades Institucionais na Resistência a Sistema de Informação.

\begin{tabular}{|c|c|}
\hline Variável & Índice \\
\hline $\begin{array}{l}\text { A cultura da organização facilita a } \\
\text { implantação de Sistemas de Informação }\end{array}$ & 0,6757 \\
\hline $\begin{array}{l}\text { O estilo de liderança dos gestores } \\
\text { contribui para a implantação de Sistemas } \\
\text { de Informação }\end{array}$ & 0,7355 \\
\hline $\begin{array}{l}\text { A organização possui regras e normas que } \\
\text { facilitam a implantação e utilização de } \\
\text { Sistemas de Informação }\end{array}$ & 0,5091 \\
\hline $\begin{array}{l}\text { As normas estabelecidas informalmente } \\
\text { facilitam a implantação se Sistemas de } \\
\text { Informação }\end{array}$ & 0,5471 \\
\hline $\begin{array}{l}\text { Observo que a organização é } \\
\text { tecnologicamente avançada e preocupada } \\
\text { com esse aspecto. }\end{array}$ & 0,3641 \\
\hline $\begin{array}{lrr}\text { A organização } & \text { investe } & \text { contínua e } \\
\text { sistematicamente } & \text { em } & \text { soluções }\end{array}$ & 0,5960 \\
\hline $\begin{array}{l}\text { tecnológicas que melhorem seu } \\
\text { desempenho }\end{array}$ & \\
\hline $\begin{array}{l}\text { Os avanços em Sistemas de Informação } \\
\text { que ocorrem na organização são } \\
\text { decorrentes de uma diretriz estabelecida } \\
\text { na alta administração, não de iniciativas } \\
\text { individuais }\end{array}$ & 0,6739 \\
\hline
\end{tabular}

Alpha de Cronbach $=0,730$.

Fonte: (Pesquisa de campo,2015).

\subsection{ANÁLISE GERAL DOS ÍNDICES SOBRE RESISTÊNCIA A SISTEMA DE INFORMAÇÃO ENTRE AS SERVIDORAS DA UFRA}

A Figura 5 representa uma visão geral sobre os índices calculados para os vetores demonstrados, e qual deles apresenta maior atuação para o desenvolvimento de comportamentos de RSI entre as servidoras da UFRA. De acordo com a Figura 03, o vetor pessoas apresenta pontuação entre 0,7000 e 1,000, o que indica a ausência de RSI. Da mesma forma, a predisposição para RSI é baixa com o índice de aceitabilidade de 0,7826 .

Entretanto, embora o índice de aceitabilidade e do vetor pessoas seja alto, eles não afastam a possibilidade de surgir o comportamento de RSI. Isso porque os índices dos vetores sistema $(0,5461)$, sóciotécnico $(0,5942)$ e político $(0,5181)$ ficaram entre 0,4001 e 0,6999, ou seja, os resultados foram intermediários, como observado anteriormente.

Assim, esses aspectos devem ser observados pela política institucional, pois como destaca Lapointe e Rivard (2005), o objeto de resistência muda conforme o avanço do tempo na implantação do sistema, passando de questões puramente técnicas para questões mais politizadas. Essas mudanças são acionadas principalmente por alterações nas condições inicias, ou seja, em como o ambiente era sobre responsabilidades, tarefas e principalmente na estrutura de poder, em relação à alteração de status, e ameaças ao nível individual ocorrida depois da implantação e as novas regras incorporadas pelo novo sistema de informação (MARKUS, 1983; JOSHI, 1991; MARAKAS E HORNIK, 1996; LAPOINTE E RIVARD, 2005; JOIA, 2006). 
Figura 3. Vetores de Resistência a Sistema de Informação.

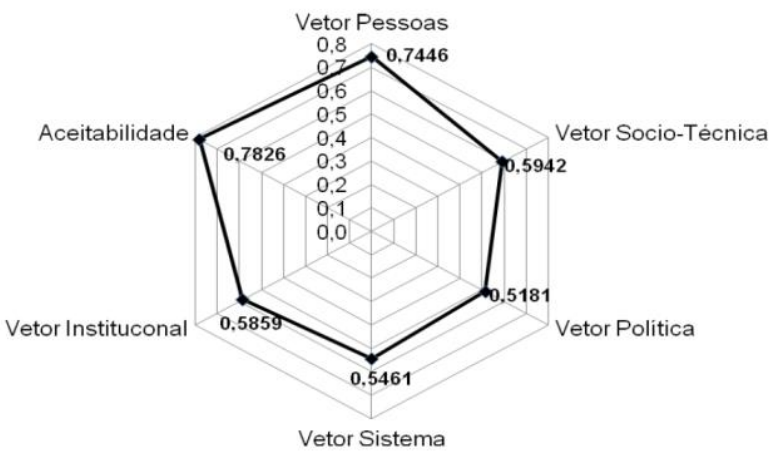

Fonte: (Pesquisa de campo, 2015).

Para tratar do grau de RSI na universidade envolvida na pesquisa, foram pré-definidos três níveis (baixo, médio e alto) para cada vetor (aceitabilidade, pessoa, sistemas, sócio-técnico, político e propriedades institucionais), bem como foi calculado um resultado geral sobre todos os vetores. $\mathrm{O}$ critério utilizado para a composição dos grupos foi estabelecido da seguinte maneira:

- Orientação Baixa $\rightarrow[\mathrm{X}]<$ (Média - 1,0.Desvio)

- Orientação Média $\rightarrow$ (Média- 1,0.Desvio) <= $[\mathrm{X}]<$ (Média + 1,0.Desvio)

- Orientação Alta $\rightarrow[X]>=$ (Média+ 1,0.Desvio). Os resultados apontados na Tabela 8 destacam que grande parte das mulheres servidoras na UFRA entrevistadas, ou seja, 89 entrevistadas (64,5\%) apresenta um grau médio de RSI, ou seja, elas não têm um alto grau de resistência, entretanto não apresentam também grande índice de aceitabilidade. Embora seja bom que apenas 20 entrevistadas apresentem alto grau de RSI $(14,5 \%)$, o percentual de entrevistadas que possuem grau baixo de RSI é pequeno também, 29 entrevistadas (21\%). O vetor sócio-técnico continua sendo um foco de destaque, relacionado principalmente com a redistribuição de tarefas e responsabilidades, apresenta maior grau de RSI entre os vetores (21\%). Os vetores pessoa, sistemas, propriedades institucionais e político, principalmente quando analisados questões como treinamento individual, usabilidade $\mathrm{e}$ organização do sistema, cultura de organização e mudança de status de pessoa, devem ser considerados como variáveis que podem futuramente influenciar num quadro

\begin{tabular}{|c|c|c|c|c|c|}
\hline \multirow[t]{2}{*}{ Vetor } & \multirow[t]{2}{*}{ Média } & \multirow{2}{*}{$\begin{array}{l}\text { Desvio } \\
\text { Padrão }\end{array}$} & \multirow{2}{*}{$\begin{array}{l}\begin{array}{l}\text { Grau } \\
(\%)\end{array} \\
\text { Baix } \\
0\end{array}$} & \multicolumn{2}{|c|}{ de Resistência } \\
\hline & & & & $\begin{array}{l}\text { Médi } \\
\text { o }\end{array}$ & Alto \\
\hline $\begin{array}{l}\text { Aceitabilida } \\
\text { de }\end{array}$ & 0,6261 & 0,1854 & 0 & 84,1 & 15,9 \\
\hline Pessoas & 0,8066 & 0,1504 & 18,1 & 65,9 & 15,9 \\
\hline Sistemas & 0,5461 & 0,2560 & 13,8 & 71,0 & 15,2 \\
\hline $\begin{array}{l}\text { Sócio- } \\
\text { Técnico }\end{array}$ & 0,5942 & 0,2315 & 10,1 & 68,8 & 21,0 \\
\hline Política & 0,5181 & 0,3295 & 19,6 & 69,9 & 19,6 \\
\hline $\begin{array}{l}\text { Propriedad } \\
\text { es } \\
\text { Instituciona } \\
\text { is }\end{array}$ & 0,5681 & 0,2201 & 21,0 & 63,8 & 15,2 \\
\hline Geral & 0,6561 & 0,1312 & 21,0 & 64,5 & 14,5 \\
\hline
\end{tabular}

maior de resistência.

Tabela 7. Grau de Resistência a Sistema de Informação

Fonte: (Pesquisa de campo, 2015).

\section{CONSIDERAÇÕES FINAIS}

As servidoras UFRA no geral apresentam resistência intermediária aos SI. Quando analisadas em relação às variáveis ligadas ao vetor pessoas a resistência é baixa, conforme foi observado nos capítulos 
anteriores. Quando as pessoas não apresentam resistência pessoal, contribuem não apenas para o bom funcionamento dos sistemas, como para sua melhoria, reconhecendo-o como uma das ferramentas para aperfeiçoar os serviços oferecidos e propor novas funcionalidades.

Entretanto, quando o fenômeno é analisado sobre os aspectos dos vetores sistemas, sócio-técnico, político e propriedades institucionais, os resultados revelam-se novamente como intermediários, não demonstrando nem alta resistência, nem alta aceitabilidade. Tal aspecto no futuro pode converter-se em processo de RSI, caso nenhuma medida seja tomada, ou os novos sistemas intensifiquem aspectos relacionado a esse tipo de comportamento.

Ao identificar os vetores que indicam processos de resistência e variáveis que contribuem para isto, acredita-se ter respondido ao questionamento central do estudo que foi "quais são as características da RSI entre as servidoras da UFRA?".

Outras observações ao longo do trabalho destacaram-se as análises das variáveis que indicaram as principais forças promotoras da existência de RSI assim como sua aceitabilidade. Esses aspectos podem minimizar consequências negativas e explorar aspectos de aceitabilidade aos SI. Embora o trabalho não tenha como objetivo identificar a ocorrência do fenômeno aos demais servidores da Instituição. A compreensão do fenômeno entre mulheres revela-se uma discussão interessante quanto a inserção das mulheres na sociedade tecnológica, onde progressivamente elas aumentam sua participação relacionada a aspectos de SI e das tecnologias de modo em geral, utilizando cada vez mais $\mathrm{Tl}$ para otimizar seus serviços e melhorar seu dia a dia, pois como foi exposto nos resultados, no vetor pessoas, elas não apresentam RSI, e admitem utilizar TI e SI para melhorar o seu dia a dia no trabalho.

\section{REFERÊNCIAS}

ALMEIDA, E. P. A Universidade como núcleo de inteligência estratégica. In: Dinossauros, Gazelas \& Tigres: novas abordagens da administração universitária. Florianópolis: Insular, 2000. p. 61-77.

ALTER, S. Information systems: a management perspective. 2. ed. Menlo Park, CA: Benjamin e Cummings, 1996.

BRASIL. Ministério do Planejamento, Orçamento e Gestão. Boletim estatístico de Pessoal, Brasília, DF, v.17, n. 200, p. 17-154, dez. 2012.

CALDAS, M. P.; HERNANDEZ, J. M. C. Resistência à mudança: uma revisão crítica. Revista de Administração de Empresas, São Paulo, n.2, v.41, p.45, abr./jun. 2001.

DUQUE, W. S.; MACHADO, M. V.; PELISARI, A.

S. Resistência à mudança tecnológica: uma análise de influências nas instâncias de clima, cultura e poder de uma empresa de importação Espírito-Santense. In: SIMPÓSIO DE EXCELÊNCIA EM GESTÃO E TECNOLOGIA,5. Anais... Resende-RJ, out. 2008.

GAETE, L. Análise da resistência a sistemas de informação: a percepção dos gestores de tecnologia da informação acerca de sistemas ERP. 2010. 137 f. Dissertação (Mestrado) Escola Brasileira de Administração Pública e de Empresas da Fundação Getúlio Vargas do Rio de Janeiro, Rio de Janeiro, 2010. HAIR, J. F. et al. Métodos de pesquisa em administração. São Paulo: Bookman, 2005. 
Joia, L. A. (2006). Lessons learnt from an unsuccessful G2G endeavor in Brazil. Proceedings of the Americas Conference on Information Systems (p. 12). Acapulco: Mexico.

JOIA, L. A.; MAGALHÃES, C. Evidências Empíricas da resistência à implantação de prescrição eletrônica: uma análise explanoexploratória. RAC-71 Eletrônica, Curitiba, v.3, n.1, art.5, p.81-104, jan./abr. 2009. Disponível em:

<http://www.anpad.org.br/rac-e>

IBGE. Censo 2010. Disponível em :http://www.ibge.gov.br/home/estatistica/p opulacao/censo2010 .Acesso em 29/12/2014.

JOSHI, K. A model of users perspective on change: the case of information systems technology implementation. MIS Quaterly, v.15, n.2, p. 229-240, June 1991.

KIM, H; KANKANHALLI, A. Investigating user resistance to information systems implementation: a status quo bias perspective. MIS Quarterly, v. 33, n. 3, p. 567-582, set. 2009.

KLING, R. Social analyses of computing: theoretical perspectives in recent empirical research. Computing Survey, v.12, n.1, p.61110, 1980.

LAPOINTE, L.; RIVARD, S. A multilevel model of resistance to information technology implementation. MIS Quarterly, v.29, n.3, p.461-491. 2005.

LAUDON, K. C.; LAUDON, J. P. Sistemas de informação com internet. 4. ed. Rio de Janeiro: Itc, 1999.

MARCONI, M. A.; LAKATOS, E. Técnicas de pesquisa. São Paulo: Atlas, 2008.

MARKUS, M. L. Power, Politics, and MIS Implementation. Massachusetts Institute of Technology, v.72, 1983.

OLINTO, G.; OLIVEIRA, Z. L. C. Gênero e trabalho precário no Brasil. Gênero, Niterói, v. 5, n.2, p. 222, 2005.

UFRA. Pró-Reitoria de Planejamento e Gestão. Superintendência de Gestão e Desenvolvimento de Pessoas. Relatório de gestão de pessoas da Universidade Federal Rural da Amazônia. Belém: UFRA, 2012.
Disponível em: $<$ http://sipac.ufra.edu.br/sigrh/public/home.j sf>. Acesso em: 29 dez. 2014.

VAZ, A. N.; MATTOS, C. A. C. Resistência aos sistemas de informação nas organizações: uma abordagem teórica. In: CONGRESSO DE PESQUISA E INOVAÇÃO DA REDE NORTE E NORDESTE DE EDUCAÇÃO TECNOLÓGICA, 6. Anais... Natal, 2011. VERGARA, S. Projetos e relatórios de pesquisa em administração. 14. ed. São Paulo: Atlas, 2013. 